\title{
Effect of Diet and Nutrients on Molecular Mechanism of Gene Expression Mediated by Nuclear Receptor and Epigenetic Modulation
}

\author{
Mahsa Jalili ${ }^{1, *}$, Sanghamitra Pati ${ }^{2}$, Bandita Rath $^{3}$, Geir Bjørklund ${ }^{4}$ and Ram B. Singh ${ }^{5}$ \\ ${ }^{I}$ Shahid Beheshti University of Medical Sciences, Tehran, Iran \\ ${ }^{2}$ Public Health Foundation of India (PHFI), Indian Institute of Public Health, Bhubaneswar, Odisha, India \\ ${ }^{3}$ Guest Faculty, Utkal University, Bhubaneswar, Odisha, India \\ ${ }^{4}$ Council for Nutritional and Environmental Medicine (CONEM), Mo i Rana, Norway \\ ${ }^{5}$ Halberg Hospital and Research Institute, Moradabad, India
}

\begin{abstract}
Major research progress in the last few decades has elucidated the complex nexus between nutrition and health. Diet and lifestyle influence epigenetic changes that are heritable. However, a statement of reservation is needed here, viz. that it is often difficult to distinguish between epigenetic changes that are inherited from one generation to the next and true mutations, for instance in mitochondrial DNA. The last topic is a big one in its own right and will not be further discussed in this article. Epigenetic changes induced by dietary nutrients ultimately culminate in changes of the expression of genes through transcription and translation. The interaction between dietary nutrients and nuclear receptors triggers the signaling pathway, leading to modulation of epigenetic change and gene expression. Knowledge about nuclear receptors is important for explaining dietary modulation of transcription via recruitment of large protein complexes. These proteins are capable of causing modification of chromosomal components, can influence chromatin proteins, and affect the binding of proteins to particular parts of the DNA molecules controlling the expression of individual genes. Chromatin complexes between DNA and proteins can be destabilized by recruitment of transcriptional coactivators by histone acetylation. However, in the presence of hormone antagonists or in the absence of relevant ligands, recruitment of other cellular core proteins may stabilize chromatin by their influence on histone deacetylases, thus antagonizing the effect of enzymes causing histone acetylation. This article reviews the current knowledge on nutritional modulation of bioactive molecules by epigenetic changes, if they can regulate genetic expressions. The molecular mechanism of action of various dietary nutrients on gene expression mediated by nuclear receptors is also discussed.
\end{abstract}

Keywords: Diet, nutrition, epigenetic changes, gene expression, transcription, nuclear receptors.

\section{INTRODUCTION}

Dietary and lifestyle factors can interact with genes and may have a negative impact of health that can predispose for the development of non-communicable diseases (NCDs), such as cardiovascular diseases (CVDs), type 2 diabetes and cancer, for which prevention is a global priority, while other nutrients and lifestyle factors can have an opposite, protective effect $[1,2]$. Recent studies conducted in the last few decades have emphasized the role of nutrition in health and prevention of NCDs. Nutrients that are available extracellularly are important determinants of eukaryotic cell growth and growth of the whole organism, since several different nutrient deficiencies can lead to inhibition or serious disturbance of growth and development at both levels. It is well established that nutrients can influence the expression of genes controlling metabolic processes via signal transduction

*Address correspondence to this author at the Nutrition Faculty, Students Research Committee, Shahid Beheshti University of Medical Sciences, Tehran, Iran; Tel: 0098 212387; E-mail: jalili.mah@gmail.com through several different transcriptional signal cascades and networks, despite still insufficient knowledge about all cellular mechanisms that mediate these effects. The body needs a prudent diet composition to be healthy without premature development of NCDs. The diet provides nutrients to various body systems that are needed for normal metabolism and optimal physiological functions, including intestinal function, cellular growth and DNA repair. The amount and quality of food has dramatic effects on processes at a molecular level in the cells.

Epigenetics is the science that aims to study quasiheritable, but reversible changes in gene function. There is no change in the nucleotide sequence of the DNA molecules; however, gene function is not only determined by the DNA sequence and code, but also by epigenetic phenomena. Recent evidence has provided further proof that environmental factors, including diet and nutrients, can influence cellsignaling pathways that control normal physiological growth as well as the growth of cancer cells by directly influencing gene expression. Epigenetic mechanisms are important determinants for activation or repression of gene expression 
[3]. The epigenetic phenomena, that involve recruitment of a wide array of chromatin remodelling complex coregulators, effectors and transcription factors finally cause remodelling of the chromatin in an appropriate manner that is, either tightly or loosely wound chromatin. DNA methylation, histone modification, and synthesis of noncoding microRNAs (miRNAs) are used as important mechanisms regulating gene expression (Figs. 1 and 2). Epigenetic alterations may be caused by nutritional factors through binding to or modification of membrane receptors upstream of signaling cascades, but also by more direct influence on gene expression by ligands binding to and activating nuclear receptors [4]. Those upstream regulatory signal mechanisms that control epigenetic alteration and further downstream signal mechanisms are thus analogous to those upstream regulatory signal mechanisms that regulate other gene expression in eukaryotic cells.

\section{DNA METHYLATION}

DNA methylation requires covalent addition of a methyl group to cytosines in eukaryotic DNA. It typically occurs at $\mathrm{CpG}$ dinucleotides. Embryonic stem cells have also non-
CpG methylation. Unmethylated CpGs are grouped in clusters, but $60-90 \%$ of all dispersed $\mathrm{CpG}$ sequences are methylated in mammals. $\mathrm{CpG}$ islands that are present in the $5^{\prime}-$ regulatory regions of many genes may also be methylated. Traditionally, it is thought that genes are suppressed because of DNA methylation.

DNA methylation is an enzymatic process. There are three enzymes, DNMT1, DNMT3a, and DNMT3b, that modify a cytosine base at the $\mathrm{CpG}$ dinucleotide residue by attaching a methyl group to form 5-methylcytosine [5, 6]. The first one, DNMT1, is a ubiquitous enzyme considered to be the major methyltransferase for maintenance methylation. It is important since it catalyzes the transfer of a methyl group from the methyl precursor, $S$-adenosyl-L-methionine (SAMe), onto the 5-position of certain cytosines in $\mathrm{CpG}$ dinucleotides. The other two DNA methylating enzymes (DNMTs) serve as de novo methyltransferases that have functions different from DNMT1. SAMe is the methyl donor in DNMT-mediated DNA methylation, as well as in several other enzymatic methylation reactions. In higher eukaryotes, DNA methylation is tissue and cell type specific.

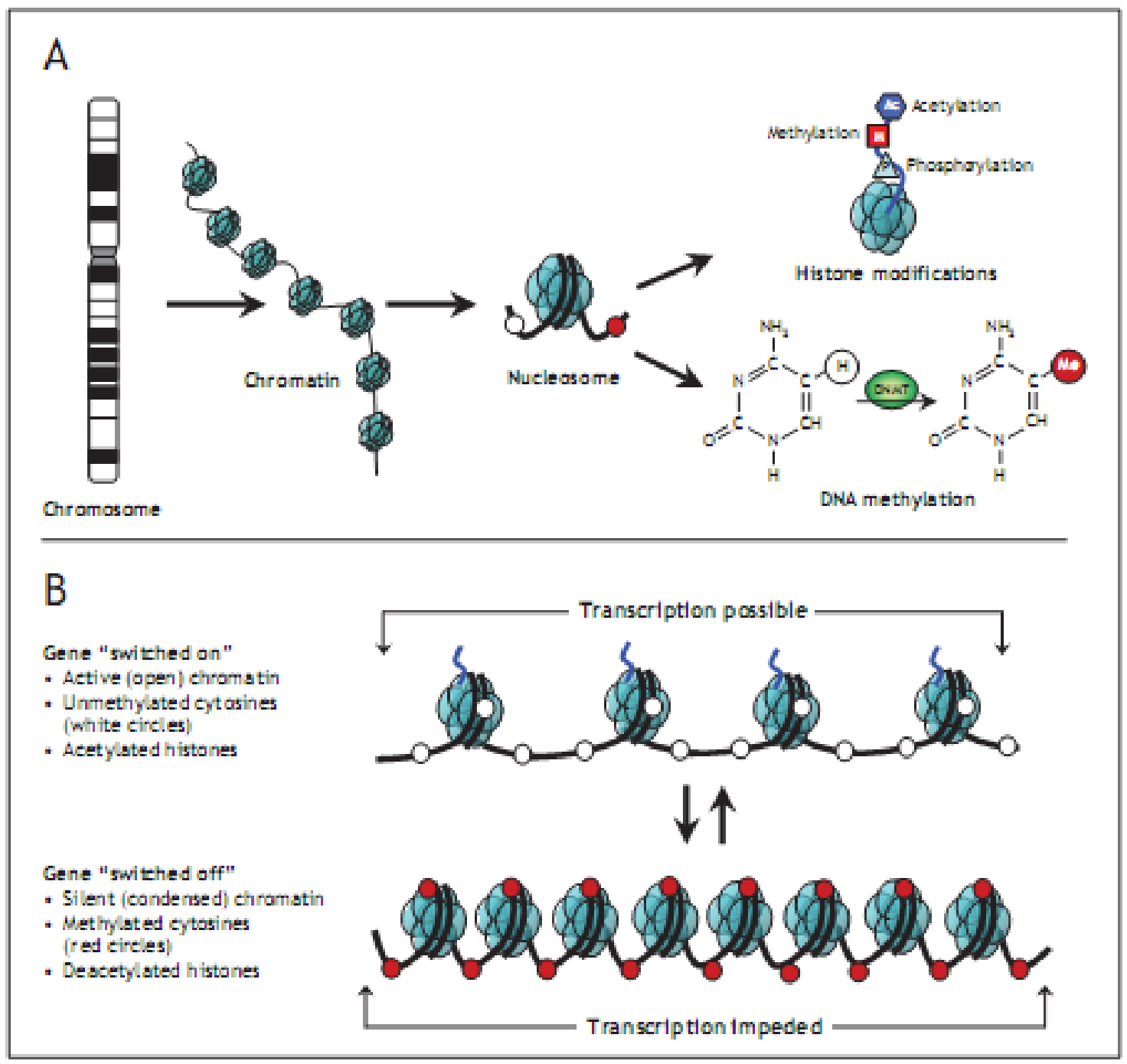

Fig.(1). Epigenetic modification on DNA strands for regulation of chromatin transcription. 


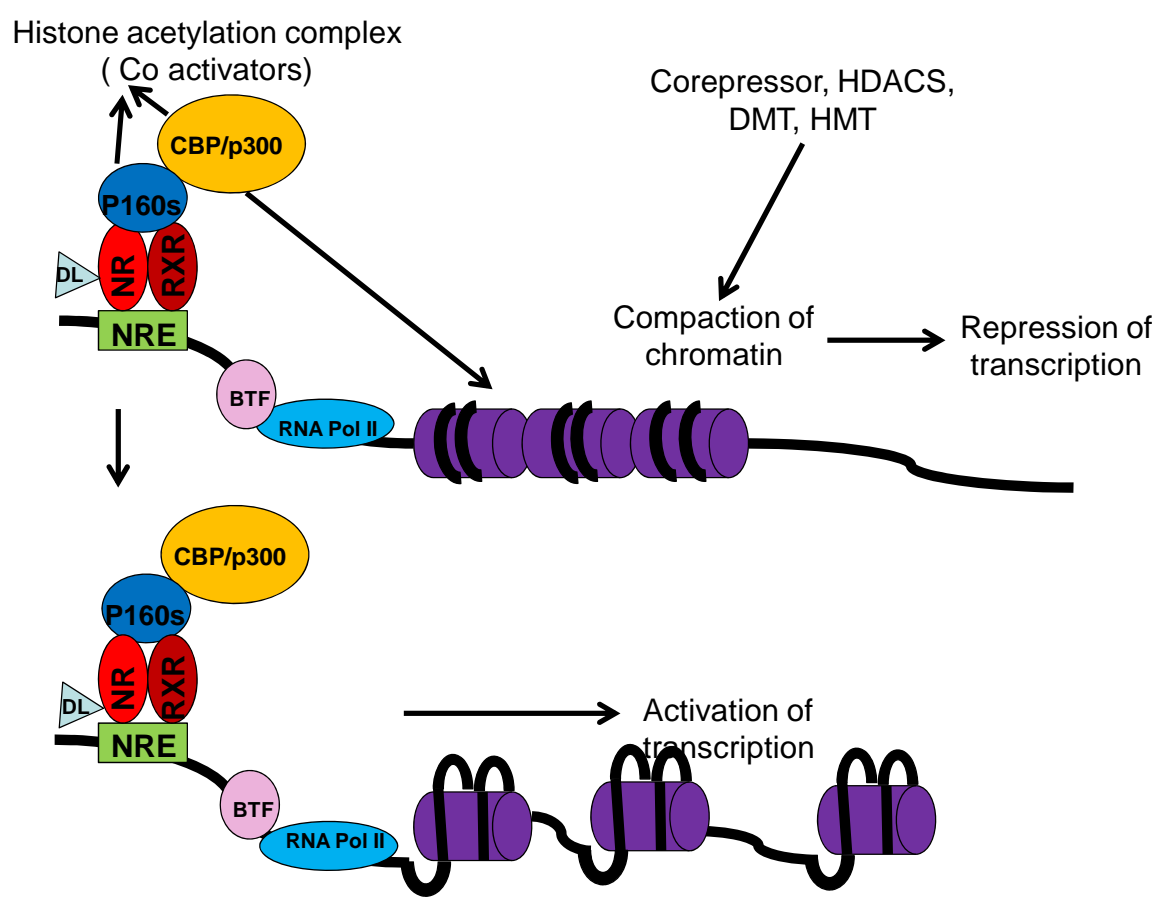

Fig.(2). The dietary factors may modulate the gene expression by nuclear receptors.

Transcriptional gene silencing may be important in the mechanisms of some diseases. In cancers, because of changes in DNMT expression and activity, gene promoter $\mathrm{CpG}$ islands may acquire abnormal hypermethylation. Cancer may thus promote DNA methylation, which may influence the transcription of genes in two major ways: First, the binding to DNA of sequence specific transcriptional factors, such as AP-2, E2F and $c$-Myc, which need the presence of unmethylated $\mathrm{CpG}$ within the binding sites, may be inhibited [7]. The other mechanism is binding to methylated DNA of proteins known as methyl-CpG-binding domain (MBD) proteins. These proteins next help in the recruitment of yet other proteins, such as histone deacetylases and also other chromatin-remodeling proteins. They are able to modify the acetylation and methylation status of histones binding to some specific genetic locus, thereby inhibiting access to the genes concerned of proteins needed for transcription. A compact, inactive chromatin may be formed subsequently [8, 9]. DNA methylation appears to be a reversible process. Therefore, its clinical aspects may be important, especially because it leads to direct silencing or indirect activation of particular genes important for progression or regression of various diseases, including cancer [10].

\section{EFFECT OF DIET AND NUTRIENTS ON DNA ME- THYLATION}

The level of DNA methylation is thus important to control gene expression to maintain the integrity of control of genome function $[11,12]$. Nutrients can have a profound effect on the level of DNA methylation, especially in genome-wide methylation. The study of these processes is an emerging field of research called nutritional genomics. Dietary deficiency of zinc (in rat liver) and selenium (in rat liver and colon) may inhibit methyl group utilization from $S$ - adenosylmethionine (SAMe), as shown especially in rat liver. This may in turn cause histone hypomethylation and genomic DNA hypomethylation [13-17]. DNA hypermethylation in lung cancer cells can occur due to vitamin $\mathrm{C}$ deficiency $[18,19]$. Niacin, by stopping the enzymatic DNA methylation, can help to maintain the unmethylated state of CpG dinucleotides [20, 21].

Inhibition of some of the enzymes participating in or controlling DNA methylation appears to be one of the important nutritional roles for niacin because it may participate in the synthesis of several factors involved $[20,21]$. However, niacin or tryptophan is also needed for the synthesis of $\mathrm{NAD}^{+}$, which is directly needed for normal DNA repair as substrate of poly(ADP-ribose) polymerase (PARP). Combined niacin and tryptophan deficiency may thus not only cause changes in the epigenetic regulation of gene expression, but also be directly mutagenic, which may be important especially in countries in sub-Saharan Africa, where maize is a major staple food.

The most compelling evidence of involvement of nutrients in DNA methylation is by folate because of its participation in one-carbon metabolism. Among water-soluble B vitamins, folate and vitamin $\mathrm{B}_{12}$ in form of methylcobalamin carry one-carbon groups. The synthesis and resynthesis of $S$ adenosyl-L-methionine (SAMe, which is also called AdoMet), the unique methyl donor for DNA methylation reactions, need folate, but also vitamin $B_{12}$ and methionine. One might thus expect dietary deficiencies of vitamin $B_{12}$ and methionine to have similar consequences as folate deficiency, and a synergistic interaction if more than one of these deficiencies are present simultaneously, which may be common in several poor countries, especially in sub-Saharan Africa. 
Methyl group donor nutrients such as folate, methionine, choline, betaine, and vitamin $\mathrm{B}_{12}$, but perhaps also omega-3 fatty acids, can alter DNA methylation. Folate has been shown to be essential for DNA methylation reprogramming during the early embryonic period. Folate deficiency in early pregnancy increases the risk of neural tube defects. Aberrant reprogramming of DNA methylation by low dietary folate has been suggested as a candidate mechanism [22]. In fact, the DNA methylation machinery is quite complex.

Reduction of promoter methylation may be mediated by resveratrol and vitamin $\mathrm{D}_{3}$. These substances downregulate DNA methyltransferase by inducing promoters of PTEN and p21. Vitamin $D_{3}$ and resveratrol have been reported to improve the inhibitory effects of $2 \mathrm{CdA}$ and F-ara-A on PTEN methylation in MCF-7 cells; however, only the combined action of vitamin $\mathrm{D}_{3}$ and $2 \mathrm{CdA}$ was found to boost the induction of PTEN expression, suggesting cooperation of these compounds in other processes driving changes in PTEN expression [23]. Bioactive food components have great potential not only in preventing, but also for treatment of cancer, cardiovascular ailments and an array of other ailments. The major target for prevention and treatment of various ailments by bioactive molecules involves alteration in DNA methylation pattern. In most cancers, hypermethylation of tumor suppressor gene leading to its inactivation is a global phenomenon.

DNMT inhibitors are important agents for cancer prevention. They can prevent as well as reverse the inactivation of key tumor suppression genes or receptor genes induced by hypermethylation. DAC and zebularine are highly toxic. Catechin, epicatechin, and epigallocatechin-3-gallate (EGCG) are polyphenols from tea. Bioflavonoids, such as quercetin, fisetin, and myricetin, as well as tea polyphenols, have been reported to inhibit DNMT- and DNMT1-mediated DNA methylation in a concentration-dependent manner. Gallic acid and EGCG the polyphenols of green tea are possible demethylating agents [5, 24]. The generation of $S$-adenosyl-homocysteine at abnormally high concentration levels is an important mechanism of inhibition of DNA methylation, as well as of other transmethylation reactions dependent on SAMe as methyl group donor. EGCG can act as a direct inhibitor of DNMT1 by the formation of hydrogen bonds with different residues in the catalytic pocket of DNMT [24-26]. Intake of polyphenols may cause a decrease in available $S$-adenosyl-L-methionine (SAMe) and an increase in $S$-adenosyl-L-homocysteine (SAH) and homocysteine levels. There is an indirect inhibition of DNA methylation by EGCG [27]. Soybeans, fava beans, lupin, kudzu, and Psoralea are important sources of genistein, being one of the isoflavones, that represent a subgroup among the flavonoid group of compounds. They have anti-cancer and antiangiogenic properties in various cancers. There is evidence that genistein has DNA methyltransferase inhibitory activity as well as causing histone modification in cancer cells. However, it is also a nonspecific inhibitor of tyrosine kinases, which can influence signal pathways controlling tumor cell growth and apoptosis at multiple loci and thus in multiple ways, and is perhaps even more important for its antitumor properties. Genistein has been reported to induce the expression of the tumor suppressor genes p21WAF1/CIP1 and
p16INK4a by altering promoter methylation and histone modification in prostate cancer cells [28, 29].

Recently, curcumin was reported to covalently block the catalytic thiolate group at the C1226 position of DNMT1, which explains its inhibitory effect by a suggested mechanism indicating interaction by molecular docking. The magnitude of this effect was nevertheless lower than for some other bioactive dietary components, such as EGCG and genistein [24, 30]. A global hypomethylation has been reported to be achieved by curcumin treatment of extracted nuclear DNA from a leukemia cell line (30). Curcumin appears to be a potent DNA hypomethylating agent, having potential inhibitory activity in inflammation, cancer, and many other chronic diseases.

Grapes, some other berries, peanuts, and other plants are important sources of resveratrol, which has also strong anticancer properties by modulating signal transduction pathways. Resveratrol influences cell division and growth, apoptosis, inflammation, angiogenesis, and metastasis, as well as helping in cancer prevention [31].

\section{MODIFICATION OF HISTONE PROTEINS}

DNA methylation is not the only mechanism important for control of the expression of genes important in cancer. Changes in the structure of chromatin can also influence gene expression in tumor cells, as well as in normal ones. This can occur by either gene inactivation, which happens when the chromatin is closed (heterochromatin), or by gene activation, which happens when the chromatin is open (euchromatin) [32]. Chromatin is the combination of DNA and small, basic histone proteins that make up much of the contents of the nucleus of a cell. DNA can only be modified by methylation. Histones, by contrast, can be modified by several kinds of reactions, such as acetylation, biotinylation, methylation, phosphorylation, ubiquitination and ADPribosylation, which are different types of chemical modifications mainly taking place within the N-terminal tails of core histones.

Acetylation of histones, which occurs at lysine residues and is catalysed by histone acetyltransferases (HATs), enhances the accessibility of the genes to the transcription machinery and activates gene transcription $[33,34]$ because the positive charge of histone molecules is reduced. Deacetylation of histones, catalysed by histone deacetylases (HDACs), makes the tail of the histone molecule highly positively charged, causing it to be tightly associated with the negatively charged DNA backbone, thus limiting the accessibility of the gene concerned to transcription factors. This will in turn lead to silencing of gene transcription $[33,35]$.

\section{NUTRIENT MODULATE GENE EXPRESSION}

Nutrients modulate gene expression by targeting transcription through nuclear receptors and HAT associated histone modifications. The basic manifestation of genetic information is the flow of information from DNA to RNA by the process called transcription. The activity of RNA polymerase is controlled by transcription factors to catalyse the formation of RNA from DNA. A transcription factor is a protein that binds to specific DNA sequences and through 
this controls the transcription of genetic information from DNA to mRNA. This function is performed by transcription factors alone or by transcription factors together with other proteins in a complex. They can act as activators by promoting or as repressors by blocking the recruitment of RNA polymerase to specific genes (Fig. 3).

Transcription factors are the main vehicles through which nutrients can influence gene expression. The nuclear hormone receptor superfamily of transcription factors, with 48 members in the human genome, is the most significant group of nutrient sensors [36-38]. The interaction between dietary nutrients and nuclear receptors triggers several signalling pathways, leading to modulation of gene expression. Recognition of more nuclear hormone receptors for their roles in the regulation of several processes, including nutrient metabolism, cell proliferation and differentiation, as well as embryonic development, remains an important unfinished scientific task.

Nutrients are capable of influencing a variety of cellular functions through activation of these receptors. Phylogenetic analysis has identified several subfamilies within this superfamily. Type I ("classical" or "steroid") receptors include those for androgens (AR), estrogens (ER), glucocorticoids (GR), mineralocorticoids (MR), and progestins (PR).

Type II receptors include those for 9-cis retinoic acid (RXR), all-trans retinoic acid (RAR), thyroid hormone (TR), and vitamin $\mathrm{D}_{3}$ (VDR). The ligands for these four receptors and their regulating pathways include elements of both the lipid-sensing and endocrine receptor pathways. Like other
RXR heterodimer ligands, retinoic acid and ecdysone are derived from essential dietary lipids (vitamin A and cholesterol, respectively), but they are not calorigenic. The transcriptional pathways that these ligands regulate (i.e. morphogenesis and development) are more similar those of the endocrine receptors. The ultimate synthesis of vitamin D and thyroid hormone and their regulating pathways are under strict endocrine control, but they need also exogenous elements for their synthesis (sunshine for vitamin $\mathrm{D}$, and iodine for thyroid hormone).

A third subclass of transcription factors contains orphan receptors, for which ligands have only recently been characterized or not at all. Although they have common structural features, the steroid and thyroid/retinoid/vitamin $\mathrm{D}_{3}$ receptor subclasses have differences in their functional characteristics. In addition, they also differ in recognition of cis-acting hormone response elements. The adopted orphan receptors respond to dietary lipids and their concentrations cannot for this cause be limited by simple negative feedback control. Members of this group include receptors for bile acids (FXR), fatty acids (PPARs), oxysterols (LXRs), and xenobiotics (steroid xenobiotic receptor or pregnane $\mathrm{X}$ receptor (SXR/PXR), and constitutive androstane receptor (CAR) [37].

Retinol is the basic form of vitamin A. It is present in foods of animal origin, where vitamin A exists in the form of retinyl esters. It can also be synthesized in the liver from $\beta$ carotene, which is present in foods of plant origin. In the body retinol becomes converted to various metabolites. They

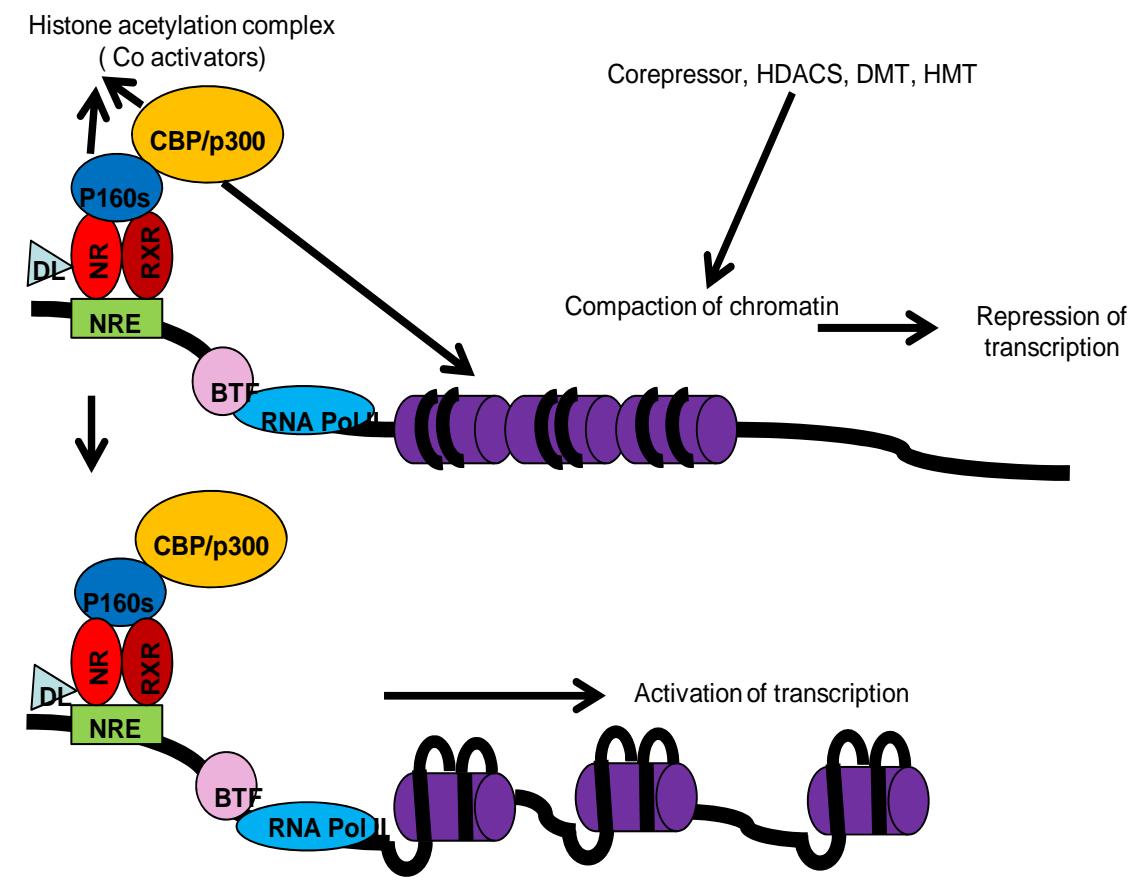

Fig. (3). Mechanism of gene regulation in response to diet:

In response to dietary ligand nuclear receptors (NRs) get recruited on nuclear receptor response element (NRE) on DNA and activate transcription by recruiting coactivator complexes, such as histone acetyltransferases (HAT) and the mediator complex, to increase chromatin accessibility by general transcription factors and promote transcription initiation. When diet induces corepressor, HDACS, DNA methyl transferase (DMT), histone methyl transferase (HMS), the nuclear and coactivator complex is dislodged from DNA and compaction of DNA occurs thus repressing transcription. 
function as signaling molecules in various biological processes, including vision, growth, metabolism, and cell differentiation. Transcriptional actions of vitamin A in vivo require the RXR/RAR heterodimer. Retinoic acid receptors (RARs) activate transcription by recruiting coactivator complexes such as histone acetyltransferases (HAT) and the mediator complex. This increases chromatin accessibility of general transcription factors and promotes transcription initiation. The pleiotropic effects of retinoids are mediated by two groups of nuclear receptors (RARs and RXRs), which are ligand-activated transcription factors. RAR/RXR heterodimers orchestrate the transcription of specific gene networks. This involves binding to specific DNA response elements and recruitment of cofactor complexes, which modifies the local chromatin structure and/or engages the basal transcription machinery. These cofactors include the p160 family (SRC-1, TIF2/GRIP1, ACTR/pCIP/RAC3/AIB1) and p300/CBP. ACTRA, p300/CBP, and SRC-1 possess HAT activity and interact with the $\mathrm{p} / \mathrm{CAF}$ histone acetyltransferase, which also interacts directly with some NRs [39-41].

The hormonally active form of vitamin $\mathrm{D}$ is 1,25dihydroxyvitamin $\mathrm{D}_{3}\left(1,25(\mathrm{OH})_{2} \mathrm{D}_{3}\right)$. It heterodimerizes with the retinoid $\mathrm{X}$ receptor and interacts with the vitamin $\mathrm{D}$ response element (VDRE) in the promoter of target genes. Vitamin D receptors exerts, similar to other steroid receptors, its transcriptional activity by interactions with proteins of the pre-initiation complex and coactivators, which may bridge the interaction between VDR and the basal transcription machinery $[42,43]$. Steroid receptor coactivators (SRCs) exhibit histone acetylase (HAT) activity. These coactivators include GR-interacting protein 1 (GRIP-1)/transcriptional intermediary factor 2 , SRC-1/NcoA1, and activator of thyroid and RARs (ACTR)/p300-CBP cointegrator-associated protein (pCIP). They can associate with other proteins that possess HAT activity, such as CREB binding protein (CBP)/p300. It is believed, as earlier explained, that HAT activity may destabilize the interaction between DNA and histone molecules, making genes accessible for DNA transcription $[44,45]$.

The peroxisome proliferator activated receptors (PPARs) are a group of nuclear receptor proteins that function as physiological lipid sensors and regulators of lipid metabolism [38]. Fatty acids and eicosanoids are natural ligands for the PPARs. More potent synthetic PPAR ligands, including thiazolidinediones and fibrates, are effective in the treatment of diabetes and dyslipidemia. PPARs and their ligands are important in various pathological states including atherosclerosis, cancer, demyelination, infertility, and inflammation. PPARs in the classical model of activation heterodimerize with RXR, and dock to PPRE (DR-1), which consists of direct repeats of AGGTCA separated by a single intervening nucleotide. Activation of transcription through this dimer is blocked by associated co-repressor proteins, such as Gprotein pathway suppressor 2 (GPS2), histone deacetylases $(\mathrm{HDAC})$, and nuclear receptor co-repressors $(\mathrm{NCoR})$. The appearance of a ligand causes dissociation of the corepressor proteins followed by the recruitment of coactivators, including PPAR coactivator (PGC-1), CREB binding protein (CBP), and histone acetyltransferase p300. Formation of the PPAR activation complex leads to histone acetylation and gives rise to altered expression of genes involved in lipid homeostasis, fatty acid metabolism, and adipocyte differentiation $[46,47]$.

Liver X receptors (LXRs) are members of the nuclear receptor superfamily of ligand-activated transcription factors. Endogenous agonists of LXRs include a variety of oxysterols, i.e. oxidized derivatives of cholesterol and phytosterols. Oxysterols can be created in the human organism through different oxidation processes, some requiring enzymes. They are also present in food due to lipid oxidation reactions caused by heating treatments, oxygen exposure, sunlight, etc. Oxysterols can be absorbed from the diet at different rates depending on their side chain length. LXRs are considered as key transcriptional regulators of lipid and carbohydrate metabolism. There are two LXR isoforms called $\alpha$ and $\beta$. Upon activation they form heterodimers with retinoid X receptor, and bind to the LXR response element in the promoter region of the target genes. This initiates recruitment of HAT coactivators, which leads to unwinding of the DNA and activation of transcription $[48,49]$.

\section{BIOACTIVE NUTRIENTS AND HISTONE MODIFI- CATION}

Dietary HDAC inhibitors are possible candidates for prevention and treatment of CVD, cancer and many other physiopathological conditions. Curcumin and EGCG have strong HAT inhibitory activity.

EGCG has been shown to abrogate p300-induced p65 acetylation both in vitro and in vivo, leading to an increased level of cytosolic IkappaBalpha, and suppresses tumor necrosis factor alpha-induced NF-kappaB activation. EGCG induces p65 deacetylation events leads to a cytoplasmic accumulation of IkappaBalpha and subsequent cytosolic sequestration of NF-kappaB that results in the downstream inhibition of inflammatory genes such as COX-2, IL-6, and NOS2 [50].

Curcumin is an effective HDAC inhibitor, and even more potent than valproic acid and sodium butyrate, which are both well-known HDAC inhibitors. It is also a potent HAT inhibitor. Curcumin is a specific inhibitor of p300/CREBbinding protein (CBP) HAT activity in vitro and in vivo, but not of p300/CBP-associated factor [51]. Curcumin inhibits p300-mediated acetylation of RelA, a subunit of NF-kappaB, which through interaction with HDAC3 is deacetylated. This deacetylation promotes effective binding to IkappaBalpha and leads to IkappaBalpha-dependent nuclear export of the complex through a chromosomal region maintenance-1dependent pathway [52]. Curcumin treatment significantly reduces HAT activity, p300 levels, and acetylated CBP/p300 gene expression, and consequently decreases NF-карраB binding [53, 54]. The ability of curcumin to inhibit p300/CBP HAT activity may be responsible, at least in part, for its potent NF-карраB inhibitory activity.

Genistein induces the expression of the tumor suppressor genes p16INK4a and p21WAF1/CIP1 in prostate cancer cells by altering promoter methylation and histone modification [28, 29]. It may also increase acetylated histones 3 and 4 and $\mathrm{H} 3$ lysine 4 at the p16INK4a and p21WAF1/CIP1 transcription start sites, mediated by induction of HATs. Genistein-mediated histone demethylation and acetylation acti- 
vates tumor suppressor genes in human prostate cancer cells, followed by cell cycle arrest and apoptosis induced by cyclin and caspase pathways [28, 29].

\section{MIRNA ASSOCIATED EPIGENETIC REGULATION MEDIATED BY DIET}

The discovery of microRNAs (miRNAs) was an extremely important breakthrough in the research and understanding of gene regulation. A microRNA (miRNA) is a small non-coding RNA molecule (ca. 22 nucleotides), which functions in the control of gene expression after transcription. Some of the epigenetic mechanisms, including DNA methylation and histone modification, regulate the expression of the protein-encoding genes, as well as of miRNAs (e.g. let-7a, miR-9, miR-34a, miR-124, miR-137, miR-148 and miR-203). Epigenetic regulators are DNA methyltransferases, histone deacetylases, and polycomb group genes. These may be under the influence of miRNA, which forms the epigenetic-miRNA regulatory circuit. Damage of this regulatory circuit disrupts normal physiological processes, which can cause various diseases.

MiRNAs have been shown to be involved in all stages of cancer, from initiation to tumor growth and progression. They influence cell proliferation, differentiation, apoptosis, angiogenesis and metastasis $[55,56]$. Recent evidence from experiments in humans and animal models suggest that nutrition (e.g., intake of vitamin E, fat, protein and alcohol) affects the expression of many miRNAs. Dietary or nutritional factors can impact miRNAs involved in various stages of carcinogenesis, including early chemoprevention versus late age therapeutic effects. All-trans-retinoic acid (vitamin A) treatment has been shown to upregulate several tumor suppressor miRNAs in human APL cells [57]. Garzon and coworkers identified putative NFkappaB consensus elements in the upstream genomic region of let-7 cluster following alltrans-retinoic acid (ATRA) treatment [57]. Cancer chemopreventive effects of vitamin $\mathrm{D}$ and its metabolites are mediated via binding with its receptor (VDR). MiR-125b has a potential sequence match in the 3'-UTR region of human VDR mRNA. This indicates a pathway for targeted therapy via VDR downregulation in human malignancies [58]. Vitamin E modulates lipid metabolism, inflammation, and other cancer-associated pathways by altering the expression of miR-122 and miR-125b [59].

Bioactive nutrients, such as curcumin, resveratrol, catechins, ellagitannin and indoles, have shown considerable downregulation in tumour marker miRNAs and a significant upregulation in tumour suppresser miRNAs, thus establishing their anti-cancer activities. Nutrients can also influence trans-generational epigenetic inheritance $[60,61]$.

\section{CONCLUSION}

The interest in diet modulated epigenetics stems from the fact that epigenetic modifications induced by diet are implicated in virtually every step of growth and development, and also in disease conditions such as tumorigenesis, CVD and diabetes. Diet-induced epigenetic modifications ultimately manifests itself through gene expressions. This review article elucidates in detail the molecular mechanism of diet-induced gene expression through epigenetic modifications and diet sensors the nuclear receptors.

Nutrients and bioactive dietary nutrients have great potential as therapeutic agents. The detailed study of molecular mechanism of gene expression by dietary compounds gives reason for great optimism regarding the therapeutic potential of dietary nutrients.

\section{CONFLICT OF INTEREST}

The authors confirm that this article content has no conflicts of interest.

\section{ACKNOWLEDGEMENTS}

None Declared.

\section{REFERENCES}

[1] McKay JA, Mathers JC. Diet induced epigenetic changes and their implications for health. Acta Physiol (Oxf) 2011; 202: 103-18.

[2] WHO/FAO. Diet, Nutrition and the Prevention of Chronic Diseases. WHO Technical Report Series 916. Geneva: World Health Organization 2003.

[3] Davis CD, Ross SA. Dietary components impact histone modifications and cancer risk. Nutr Rev 2007; 65: 88-94.

[4] Gabory A, Attig L, Junien C. Sexual dimorphism in environmental epigenetic programming. Mol Cell Endocrinol 2009; 304: 8-18.

[5] Meeran SM, Ahmed A, Tollefsbol TO. Epigenetic targets of bioactive dietary components for cancer prevention and therapy. Clin Epigenet 2010; 1: 101-16.

[6] Bestor T. The DNA methyltransferases of mammals. Hum Mol Genet 2000; 9: 2395-402.

[7] Tate P, Bird A. Effects of DNA methylation on DNA-binding proteins and gene expression. Curr Opin Genet Dev 1993; 3: 22631.

[8] Wade P. Methyl CpG-binding proteins and transcriptional repression. Bioessays 2001; 23: 1131-7.

[9] Ballestar E, Wolffe A. Methyl-CpG-binding proteins. Targeting specific gene repression. Eur J Biochem 2001; 268: 1-6.

[10] Laird P. Cancer epigenetics. Hum Mol Genet 2005; 14 (Spec No 1): R65-76.

[11] Wolffe AP, Matzke MA. Epigenetics: regulation through repression. Science 1999; 286: 481-6.

[12] Jones PA, Laird PW. Cancer epigenetics comes of age. Nat Genet 1999; 21: 163-7.

[13] Wallwork JC, Duerre JA. Effect of zinc deficiency on methionine metabolism, methylation reactions and protein synthesis in isolated perfused rat liver. J Nutr 1985; 115: 252-62.

[14] Dreosti IE. Zinc and the gene. Mutat Res 2001; 475: 161-7.

[15] Davis CD, Uthus EO, Finley JW. Dietary selenium and arsenic affect DNA methylation in vitro in Caco-2 cells and in vivo in rat liver and colon. J Nutr 2000; 130: 2903-9.

[16] Friso S, Choi SW. Gene-nutrient interactions and DNA methylation. J Nutr 2002; 132 (8 Suppl): 2382S-7S.

[17] El-Bayoumy K. The protective role of selenium on genetic damage and on cancer. Mutat Res 2001; 475: 123-39.

[18] Piyathilake CJ, Bell WC, Johanning GL, Cornwell PE, Heimburger DC, Grizzle WE. The accumulation of ascorbic acid by squamous cell carcinomas of the lung and larynx is associated with global methylation of DNA. Cancer 2000; 89: 171-6.

[19] Halliwell B. Vitamin C and genomic stability. Mutat Res 2001; 475: 29-35.

[20] Zardo G, Caiafa P. The unmethylated state of $\mathrm{CpG}$ islands in mouse fibroblasts depends on the poly(ADP-ribosyl)ation process. J Biol Chem 1998; 273: 16517-20.

[21] Hageman GJ, Stierum RH. Niacin, poly(ADP-ribose) polymerase-1 and genomic stability. Mutat Res 2001; 475: 45-56.

[22] Steegers-Theunissen RP, Smith SC, Steegers EA, Guilbert LJ, Baker PN. Folate affects apoptosis in human trophoblastic cells. BJOG 2000; 107: 1513-5. 
[23] Stefanska B, Salamé P, Bednarek A, Fabianowska-Majewska K. Comparative effects of retinoic acid, vitamin $\mathrm{D}$ and resveratrol alone and in combination with adenosine analogues on methylation and expression of phosphatase and tensin homologue tumour suppressor gene in breast cancer cells. Br J Nutr 2012; 107: 781-90.

[24] Fang M, Chen D, Yang CS. Dietary polyphenols may affect DNA methylation. J Nutr 2007; 137 (1 Suppl): 223S-8S.

[25] Fang MZ, Wang Y, Ai N, et al. Tea polyphenol (-)-epigallocatechin-3-gallate inhibits DNA methyltransferase and reactivates methylation-silenced genes in cancer cell lines. Cancer Res 2003; 63: 7563-70.

[26] Lee WJ, Shim JY, Zhu BT. Mechanisms for the inhibition of DNA methyltransferases by tea catechins and bioflavonoids. Mol Pharmacol 2005; 68: 1018-30.

[27] Lee WJ, Zhu BT. Inhibition of DNA methylation by caffeic acid and chlorogenic acid, two common catechol-containing coffee polyphenols. Carcinogenesis 2006; 27: 269-77.

[28] Majid S, Kikuno N, Nelles J, et al. Genistein induces the p21WAF1/CIP1 and p16INK4a tumor suppressor genes in prostate cancer cells by epigenetic mechanisms involving active chromatin modification. Cancer Res 2008; 68: 2736-44.

[29] Kikuno N, Shiina H, Urakami S, et al. Genistein mediated histone acetylation and demethylation activates tumor suppressor genes in prostate cancer cells. Int J Cancer 2008; 123: 552-60.

[30] Liu H, Chen Y, Cui G, Zhou J. Curcumin, a potent anti-tumor reagent, is a novel histone deacetylase inhibitor regulating B-NHL cell line Raji proliferation. Acta Pharmacol Sin 2005; 26: 603-9.

[31] Bishayee A. Cancer prevention and treatment with resveratrol: from rodent studies to clinical trials. Cancer Prev Res (Phila) 2009; 2: 409-18.

[32] Rodenhiser D, Mann M. Epigenetics and human disease: translating basic biology into clinical applications. CMAJ 2006; 174: 3418 .

[33] Kondo Y. Epigenetic cross-talk between DNA methylation and histone modifications in human cancers. Yonsei Med J 2009; 50: $455-63$.

[34] Bannister AJ, Kouzarides T. Regulation of chromatin by histone modifications. Cell Res 2011; 21: 381-95.

[35] Link A, Balaguer F, Goel A. Cancer chemoprevention by dietary polyphenols: promising role for epigenetics. Biochem Pharmacol 2010; 80: 1771-92.

[36] Mangelsdorf DJ, Thummel C, Beato M, et al. The nuclear receptor superfamily: the second decade. Cell 1995; 83: 835-9.

[37] Wang K, Chen S, Xie W, Wan YJ. Retinoids induce cytochrome $\mathrm{P}_{450} 3 \mathrm{~A} 4$ through RXR/VDR-mediated pathway. Biochem Pharmacol 2008; 75: 2204-13.

[38] Müller M, Kersten S. Nutrigenomics: goals and strategies. Nat Rev Genet 2003; 4: 315-22.

[39] Lemon BD, Freedman LP. Nuclear receptor cofactors as chromatin remodelers. Curr Opin Genet Dev 1999; 9: 499-504.

[40] McKenna NJ, Lanz RB, O’Malley BW. Nuclear receptor coregulators: cellular and molecular biology. Endocr Rev 1999; 20: 321-44.

[41] Glass CK, Rosenfeld MG. The coregulator exchange in transcriptional functions of nuclear receptors. Genes Dev 2000; 14: 121-41.

[42] Di Croce L, Okret S, Kersten S, et al. Steroid and nuclear receptors. Villefranche-sur-Mer, France, May 25-27, 1999. EMBO J 1999; 18: 6201-10.
[43] Rachez C, Freedman LP. Mechanisms of gene regulation by vita$\min \mathrm{D}_{3}$ receptor: a network of coactivator interactions. Gene 2000; 246: 9-21.

[44] McKenna NJ, Xu J, Nawaz Z, Tsai SY, Tsai MJ, O’Malley BW. Nuclear receptor coactivators: multiple enzymes, multiple complexes, multiple functions. J Steroid Biochem Mol Biol 1999; 69: 3-12.

[45] Freedman LP. Increasing the complexity of coactivation in nuclear receptor signaling. Cell 1999; 97: 5-8.

[46] Barish GD. Peroxisome proliferator-activated receptors and liver X receptors in atherosclerosis and immunity. J Nutr 2006; 136: 690-4.

[47] Bordoni A, Di Nunzio M, Danesi F, Biagi PL. Polyunsaturated fatty acids: From diet to binding to PPARs and other nuclear receptors. Genes Nutr 2006; 1: 95-106.

[48] Otaegui-Arrazola A, Menéndez-Carreño M, Ansorena D, Astiasarán I. Oxysterols: A world to explore. Food Chem Toxicol 2010; 48: 3289-303.

[49] Baranowski M. Biological role of liver X receptors. J Physiol Pharmacol 2008; 59 (Suppl 7): 31-55.

[50] Choi KC, Jung MG, Lee YH, et al. Epigallocatechin-3-gallate, a histone acetyltransferase inhibitor, inhibits EBV-induced B lymphocyte transformation via suppression of RelA acetylation. Cancer Res 2009; 69: 583-92.

[51] Balasubramanyam K, Varier RA, Altaf M, et al. Curcumin, a novel p300/CREB-binding protein-specific inhibitor of acetyltransferase, represses the acetylation of histone/nonhistone proteins and histone acetyltransferase-dependent chromatin transcription. J Biol Chem 2004; 279: 51163-71.

[52] Chen Lf, Fischle W, Verdin E, Greene WC. Duration of nuclear NF-kappaB action regulated by reversible acetylation. Science 2001; 293: 1653-7.

[53] Yun JM, Jialal I, Devaraj S. Epigenetic regulation of high glucoseinduced proinflammatory cytokine production in monocytes by curcumin. J Nutr Biochem 2011; 22: 450-8.

[54] Reuter S, Gupta SC, Park B, Goel A, Aggarwal BB. Epigenetic changes induced by curcumin and other natural compounds. Genes Nutr 2011; 6: 93-108.

[55] Parasramka MA, Ho E, Williams DE, Dashwood RH. MicroRNAs, diet, and cancer: new mechanistic insights on the epigenetic actions of phytochemicals. Mol Carcinog 2012; 51: 213-30.

[56] Sato F, Tsuchiya S, Meltzer SJ, Shimizu K. MicroRNAs and epigenetics. FEBS J 2011; 278: 1598-609.

[57] Garzon R, Pichiorri F, Palumbo T, et al. MicroRNA gene expression during retinoic acid-induced differentiation of human acute promyelocytic leukemia. Oncogene 2007; 26: 4148-57.

[58] Mohri T, Nakajima M, Takagi S, Komagata S, Yokoi T. MicroRNA regulates human vitamin D receptor. Int J Cancer 2009 125: 1328-33.

[59] Gaedicke S, Zhang X, Schmelzer C, et al. Vitamin E dependent microRNA regulation in rat liver. FEBS Lett 2008; 582: 3542-6.

[60] Alam SE, Singh RB, Gupta S, et al. Nutritional aspects of epigenetic inheritance. Can J Physiol Pharmacol 2012; 90: 989-94.

[61] Mishra S, Singh RB, Dwivedi SP, et al. Effects of nutraceuticals on genetic expressions. Open Nutraceuticals J 2009; 2: 70-80.

Received: January 09, 2013

Revised: April 13, 2013

Accepted: April 16, 2013

(C) Jalili et al.; Licensee Bentham Open.

This is an open access article licensed under the terms of the Creative Commons Attribution Non-Commercial License (http://creativecommons.org/licenses/by-nc/3.0/) which permits unrestricted, non-commercial use, distribution and reproduction in any medium, provided the work is properly cited. 